\title{
Possible experiment to check the reality of a nonequilibrium temperature
}

\author{
D. Jou and J. Casas-Vázquez \\ Departament de Física (Física Estadística), Universitat Autonoma de Barcelona, 08193 Bellaterra, Catalonia, Spain
}

(Received 25 November 1991)

\begin{abstract}
An experiment is proposed to check the physical reality of a nonequilibrium absolute temperature previously proposed from theoretical grounds in the framework of extended irreversible thermodynamics.
\end{abstract}

PACS number(s): 05.70.Ln, 44.10. $+\mathrm{i}$

Some of the most basic questions in nonequilibrium thermodynamics are those concerning the fundamental thermodynamic concepts, namely, the definition and meaning of entropy and of absolute temperature out of equilibrium. Most of the nonequilibrium thermodynamic theories have assumed that these concepts do not need any reformulation out of equilibrium, and that the usual equilibrium concepts may be applied locally in nonhomogeneous nonequilibrium situations.

For some years, we have proposed a nonequilibrium absolute temperature [1-4] stemming as the partial derivative of a nonequilibrium entropy that incorporates as variables the dissipative fluxes, in the framework of the so-called extended irreversible thermodynamics (EIT) [5-10]. Up to now, we have analyzed such a nonequilibrium temperature from a theoretical point of view, either from a formal point of view involving dissipative Carnot cycles [2] or, from a more specific point of view, by studying some of the possible indirect consequences of such a generalized temperature in the propagation of heat waves in nonequilibrium steady states $[3,4]$ or in the apparent thermal conductivity in Poiseuille phonon flow [4].

The purpose of this paper is to suggest an experiment to directly check the physical reality of such a nonequilibrium temperature. First of all, we review the introduction of the generalized temperature; afterwards, we suggest the experiment and, finally, we evaluate the order of magnitude of the expected effects.

We recall that the standard thermodynamic definition of the absolute temperature is

$$
T^{-1}=\left[\frac{\partial s}{\partial u}\right]_{v},
$$

with $s$ the entropy (or the specific entropy per unit mass), $u$ the internal energy (or specific internal energy per unit mass), and $v$ the volume (or specific volume per unit mass).

The generalized entropy arising in extended irreversible thermodynamics for a rigid heat conductor has the following form

$$
s(u, \mathbf{q})=s_{\text {eq }}(u)-\left(v \tau / 2 \lambda T^{2}\right) \mathbf{q} \cdot \mathbf{q},
$$

where $s_{\text {eq }}(u)$ is the local-equilibrium entropy, $T$ the local-equilibrium absolute temperature, $q$ the heat flux, $\lambda$ the thermal conductivity, and $\tau$ the relaxation time of the heat flux, defined according to the relaxational evolution equation

$$
\tau\left(\frac{\partial \mathrm{q}}{\partial t}\right)+\mathrm{q}=-\lambda \nabla \Theta,
$$

where the quantity $\Theta$ is a generalized absolute temperature defined according to (1), i.e.,

$$
\Theta^{-1}=T^{-1}-\gamma \mathbf{q} \cdot \mathbf{q},
$$

with $\gamma=\partial\left(v \tau / 2 \lambda T^{2}\right) / \partial u$.

An equation of the form (3), with $T$ instead of $\Theta$, is known as Maxwell-Cattaneo-Vernotte equation, and has been used in several occasions to describe heat waves in solids at low temperatures [11]. For a detailed discussion of this equation and of its thermodynamic consequences, the reader is referred to [5(b)]. The appearance of $\Theta$ instead of $T$ is imposed by thermodynamic requirements which are a direct consequence of the use of the generalized entropy (2) instead of the local-equilibrium entropy, and which are examined at length in [3-5]. It must be noted that EIT does not pretend to be an exact theory, but rather a model which allows for formulation of a nonequilibrium thermodynamic theory not based $a$ priori on the local-equilibrium hypothesis, and which allows for exploration of the limitations of this hypothesis and for new questions which are out of the scope of the classical theory.

It must be pointed out that a nonequilibrium absolute temperature is a concept that does not arise in the classical local-equilibrium theory [12-13], but that is not exclusive of extended irreversible thermodynamics. For instance, different concepts of "nonequilibrium temperatures" have been considered in other contexts by other authors, such as Meixner (who sets the concept of a "dynamical temperature" [14]), Müller (who introduces a "coldness" [15]), Muschick (who postulates a "contact temperature" [16]), and Keizer (in the framework of his formulation of nonequilibrium thermodynamics based on statistical considerations of molecular fluctuations $[17,18])$. To our knowledge, none of these abstract temperatures has had any direct experimental confirmation. As compared with these other temperatures, the nonequilibrium temperature of extended thermodynamics and that of Keizer have the advantage of having an explicit expression allowing for an evaluation of the nonclassi- 
cal effects.

In order to check whether the quantity $\Theta$ defined in (4) is a mathematical artifact arising from an undue extension of the classical definition (1), or whether it is an actual physical quantity, we propose the following experiment. We connect by means of a plate of a good thermal conductor (such as, for instance, $\mathrm{Cu}$ or $\mathrm{Ag}$ ) two thermodynamic systems. One of such systems is at equilibrium, whereas the other one is in a nonequilibrium steady state characterized by a heat flux $q_{y}$ perpendicular to the connection between both systems (Fig. 1). Such a connection is installed in such a form that the local-equilibrium temperature of the nonequilibrium system at the position of the connection is equal to the temperature of the equilibrium system. According to (3), a heat flux $q_{x}$ should be observed between both systems. Such a flux would be given by

$$
q_{x}=\lambda_{m}(\Theta-T) / l,
$$

where $\lambda_{m}$ is the thermal conductivity of the metallic plate providing the connection between both systems and $l$ is the separation between them. In contrast, in the local-equilibrium theory $q_{x}$ should be given as $q_{x}=\lambda_{m}(T-T) / l=0$, because we have assumed that both ends of the connection are at the same localequilibrium temperature $T$.

According to (4), the heat flux $q_{x}$ should be, up to the second order in $q_{y}$,

$$
q_{x}=\left(\lambda_{m} / l\right) \gamma T^{2} q_{y}^{2}
$$

The presence of such a heat flux $q_{x}$ as predicted in (6) would be a strong support to the physical reality of the generalized absolute temperature $\Theta$.

Finally, we proceed to make an estimation of the order of magnitude of the predicted nonclassical effect. For an ideal gas and for a metallic conductor, the explicit expressions of the generalized temperature (4) are given by

$$
\begin{aligned}
& \Theta^{-1}=T^{-1}+(2 / 5)\left(m / n^{2} k^{3} T^{4}\right) \mathbf{q} \cdot \mathbf{q}, \\
& \Theta^{-1}=T^{-1}+\left(9 / \pi^{4}\right)\left(m \varepsilon_{F} / n^{2} k^{4} T^{5}\right) \mathbf{q} \cdot \mathbf{q},
\end{aligned}
$$

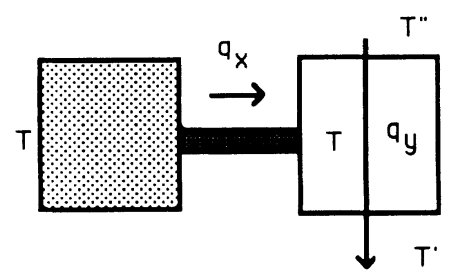

FIG. 1. Two thermodynamic systems are connected through a highly conducting metallic plate. The system on the left is at equilibrium at temperature $T$. The system on the right is in a nonequilibrium steady state under a heat flux $q_{y}$. Both ends of the conducting plate are at the same local-equilibrium absolute temperature $T$. According to the classical theory, no heat should flow from one system to the other one. In contrast, EIT predicts a flow $q_{x}$ from the left to the right because the nonequilibrium thermodynamic temperature of the right-hand side system, $\Theta$, is not equal to the local-equilibrium absolute temperature $T$. where $m$ is the mass of the molecules in (7) or the electron mass in (8), $n$ is the number particle density, $\varepsilon_{F}$ is the Fermi energy of the metal, and $k$ is the Boltzmann constant $\left(k=1.38 \times 10^{-23} \mathrm{~J} / \mathrm{K}\right)$.

To estimate $q_{x}$, assume, for instance, that the subsystems are composed of $\mathrm{CO}_{2}$ at $300 \mathrm{~K}$ and $0.1 \mathrm{~atm}$. Then, $m=4 \times 10^{-26} \mathrm{~kg}$ and $n=2.6 \times 10^{24}$ particles $/ \mathrm{m}^{3}$, and we have for (6) $q_{x}=\left(\lambda_{m} / l\right)\left(9.6 \times 10^{-12}\right) q_{y}^{2}$, with $q_{y}$ expressed in $\mathrm{W} / \mathrm{m}^{2}$. Thus, for $q_{y}$ of the order of $10^{5} \mathrm{~W} / \mathrm{m}^{2}$, the effective temperature difference $\Theta-T$ would be of the order of $9.6 \times 10^{-2} \mathrm{~K}$. We think it would be better to measure $q_{x}$ rather than $\Theta-T$ itself because of the conceptual difficulties in the definition and measurement of temperature in nonequilibrium. Note that to achieve $q_{y}=10^{5}$ $\mathrm{W} / \mathrm{m}^{2}$ one would need a temperature gradient in the $y$ direction of the order of $10^{4} \mathrm{~K} / \mathrm{m}$ because the thermal conductivity of the gas is of the order of $10 \mathrm{~W} / \mathrm{K} \mathrm{m}$. Such a temperature gradient is easy to achieve. At such conditions, and using a copper plate $\left(\lambda_{m}=3.8 \times 10^{2}\right.$ $\mathrm{W} / \mathrm{K} \mathrm{m}$ ) of $1 \mathrm{~cm}$ in length to connect both systems, one would have $q_{x}=3.65 \times 10^{3} \mathrm{~W} / \mathrm{m}^{2}$, which should be perceptible.

If the two subsystems are made of copper $\left[n=8.45 \times 10^{22} \quad \mathrm{~cm}^{-3}, \quad T_{F}=\varepsilon_{F} / k=8.12 \times 10^{4} \mathrm{~K}\right.$, $m=9.1 \times 10^{-31} \mathrm{Kg}$, the difference $\Theta-T$ for a temperature gradient in the $y$ direction of the order of $10^{4} \mathrm{~K} / \mathrm{m}$ would be of the order of $2 \times 10^{-3} \mathrm{~K}$, less than for the gas of the previous example. In this situation, the heat-flux $q_{x}$ due to the difference $\Theta-T$ would be $q_{x}=76 \mathrm{~W} / \mathrm{m}^{2}$.

It is worthwhile to point out that the entropy (2) is confirmed from kinetic theory of gases in the 13-moment Grad's approximation [19]. More general expressions for $s$ as a function of $q$ may be found in more sophisticated developments in kinetic theory [20]. But in kinetic theory of monatomic gases the temperature is identified through the expression $u^{\prime}=(3 / 2) k T, u^{\prime}$ being the mean kinetic energy per molecule, whereas the thermodynamic definition (1) is not used. Our suggested experiment could contribute to clarify the physical significance of both temperatures. Thus the interest of such an experiment is not restricted to extended irreversible thermodynamics, but may be of more general interest in nonequilibrium thermodynamics and statistical mechanics.

The relation between the generalized temperature of extended thermodynamics and that of Keizer deserves some special mention. In fact, both temperatures are different than the local-equilibrium absolute temperature, and both depend on the fluxes besides on the classical variables. The difference between both theories is found in their respective starting points: extended thermodynamics is motivated by the dynamics of the fluxes considered as independent variables; Keizer's theory has its origin in the second moments of nonequilibrium fluctuations. It would be of interest to explore whether both theories lead to a same expression for the nonequilibrium temperature in a given definite situation: it is likely to expect a confluence of both theories in this point. One must note, however, that the experiment we are proposing would lead to the conclusion that a thermometer does not measure the local-equilibrium temperature; but the 
generalized temperature, because the heat exchange between the system and the thermometer is related to differences in the latter one rather than to differences in the local-equilibrium temperature, according to extended thermodynamics. This point of view is different from that of Keizer, who assumes [17] that the thermometer reads the local-equilibrium temperature and that the effects of the nonequilibrium temperature would be seen through an analysis of nonequilibrium fluctuations.

Temperature would not be the only thermodynamic quantity to contain nonequilibrium corrections: they would also arise in the pressure or in the chemical potential. Keizer has studied theoretically and experimentally the corrections to the electromotive force in a nonequilibrium situation [21]. The experimental consequences of the nonequilibrium contributions to the chemical potential in polymeric solutions have been studied in the framework of extended irreversible thermodynamics [22]. In both analyses, the corrections turn out to be measurable. However, the corrections have been more elusive in the analysis of temperature. This is not surprising, as the measurement of temperature in nonequilibrium steady states is still an open problem. Though our experiment does not set a completely general nonequilibrium temperature, as it is restricted to situations where the heat flux is the only dissipative quantity, it is evident that the confirmation of the physical reality of a nonequilibrium absolute temperature would be a very important fact in nonequilibrium thermodynamics, either from a theoretical point of view concerning the foundations themselves of irreversible thermodynamics, or from an experimental point of view, since intense heat fluxes may be found in actual physical systems as, for instance, in the sun and other stars, in gravitational collapse, in explosions, or in implosions of hydrogen pellets in laser-induced fusion reactions.

This work has been supported by the Dirección General de Investigación Científica y Tecnológica of the Spanish Ministry of Education and Science under Grant No. PB-90/676. We acknowledge stimulating discussions with Professor G. Lebon, from Liège University, and Professor J. J. Brey, from Sevilla University.
[1] J. Casas-Vázquez and D. Jou, J. Phys. A 14, 1221 (1981)

[2] D. Jou and J. Casas-Vázquez, J. Phys. A 20, 5371 (1988).

[3] J. Casas-Vázquez and D. Jou, Acta Phys. Acad. Sci. Hung. 66, 99 (1989).

[4] D. Jou and J. Casas-Vázquez, Physica A 163, 47 (1990).

[5] (a) Recent Developments in Nonequilibrium Thermodynamics, edited by J. Casas-Vázquez, D. Jou, and G. Lebon (Springer, Berlin, 1984); (b) D. Jou, J. Casas-Vázquez, and G. Lebon, Rep. Prog. Phys. 51, 1105 (1988); (c) G. Lebon, D. Jou, and J. Casas-Vázquez, J. Phys. A 13, 275 (1980).

[6] L. S. García-Colín, Rev. Mex. Fís. 34, 344 (1988); L. S. García-Colín, M. López de Haro, R. F. Rodríguez, J. Casas-Vázquez, and D. Jou, J. Stat. Phys. 37, 465 (1984); L. S. García-Colín, R. Luzzi, and A. Vasconcellos, Phys. Rev. A 43, 6622 (1991); 43, 6633 (1991).

[7] R. E. Nettleton, Phys. Fluids 3, 216 (1960); J. Phys. A 20, 4017 (1990).

[8] I. Müller, Z. Phys. 198, 329 (1967); Thermodynamics (Pitman, London, 1985).

[9] B. C. Eu, J. Chem. Phys. 73, 2958 (1980).

[10] Advances in Thermodynamics: Nonequilibrium Thermodynamics, edited by S. Sieniutycz and P. Salamon (Taylor and Francis, New York, 1992).
[11] D. D. Joseph and L. Preziosi, Rev. Mod. Phys. 61, 41 (1989).

[12] S. R. de Groot and P. Mazur, Nonequilibrium Thermodynamics (North-Holland, Amsterdam, 1962).

[13] P. Glansdorff and I. Prigogine, Thermodynamic Theory of Structure, Stability and Fluctuations (Wiley, New York, 1971).

[14] J. Meixner, in Foundations of Continuum Thermodynamics, edited by J. J. D. Domingos, M. N. R. Nina, and J. H. Whitelaw (MacMillan, London, 1974).

[15] I. Müller, Arch. Ration. Mech. Anal. 40, 1 (1971).

[16] W. Muschick, Arch. Ration. Mech. Anal. 66, 379 (1977); J. Non-Equilib. Thermodyn. 4, 277 (1979).

[17] J. Keizer, J. Chem. Phys. 65, 4431 (1976); 69, 2609 (1978); 82, 2751 (1985).

[18] J. Keizer, Statistical Thermodynamics of Nonequilibrium Processes (Springer, New York, 1987).

[19] H. Grad, in Principles of the Kinetic Theory of Gases, edited by S. Flugge, Handbuch der Physik Vol. XII (Springer, Berlin, 1958).

[20] A. Santos and J. J. Brey, Physica A 174, 355 (1991).

[21] J. Keizer, J. Chem. Phys. 87, 4064 (1987); 87, 4074 (1987).

[22] M. Criado-Sancho, D. Jou, and J. Casas-Vázquez, Macromolecules 24, 2834 (1991). 